\title{
ELECTROMODULATED ABSORPTION IN SMOKY QUARTZ
}

\author{
W. E. VAN DEN BROM and J. VOLGER \\ Fysisch Laboratorium der Rijksuniversiteit Utrecht, Afdeling Vaste Stof Fysica, \\ Utrecht, Nederland
}

Received 26 February 1974

\section{Synopsis}

The optical absorption coefficient of "smoky" quartz (containing aluminium) can be modulated by applying an electric field. The effect saturates at high fields and low temperatures and reaches a maximum at $535 \mathrm{~nm}$. The results are discussed in terms of a model consisting of a colour centre, dipolar in character and exhibiting anisotropic absorption. Realignment of the dipoles induces changes in the observed absorption. The experiments suggest that the centre is an $\mathrm{XO}_{4}$ complex with a hole trapped at one of the oxygens, and that $\mathrm{X}$ is an ion substituted for a $\mathrm{Si}^{4+}$ ion. $\mathrm{X}$ cannot be identified with the well-known aluminium impurity; it is tentatively proposed to be an iron ion (as in amethyst).

1. Introduction. During the past decade much attention has been paid to dipole centres in solids. A large part of the investigations has been devoted to "ionic" dipoles, mainly incorporated in alkali-halide host lattices and consisting of di- or triatomic molecules (like $\left.\mathrm{OH}^{-}\right)^{1}$ ), or of ions occupying an off-centred position $\left.{ }^{2}\right)$ (like $\mathrm{Li}^{+}$in $\left.\mathrm{KCl}\right)$. "Electronic" dipoles form another class of dipole centres. They may arise when electrons (or holes) are trapped in the neighbourhood of charged impurities (like $\mathrm{Li}^{+}$in $\left.\mathrm{NiO}\right)^{3}$ ). In both cases orientationally degenerate states may occur and reorientation of the dipole is in principle possible by hopping over or tunneling through the potential barriers, separating the equilibrium positions of the molecule or the electron (or hole), respectively. The literature on electronic dipole centres is not very extensive as compared with that on ionic dipole centres. Besides the case of $\mathrm{NiO}: \mathrm{Li}$ we mention the electronic dipole centres in reduced rutile $\left.\left(\mathrm{TiO}_{2}\right)^{4}\right)$ and in $\mathrm{X}$-irradiated smoky quartz ${ }^{5,6}$ ).

Direct observation of the mechanism of alignment is possible by means of dielectric relaxation experiments and the counter part of paramagnetic resonance, paraelectric resonance.

In some cases the reorientation process may also be observed in an 
indirect way, if, in addition, the dipole centre possesses some other specific, for instance paramagnetic or optical, properties. A well-known example is the experiment of Taylor and Farnell ${ }^{7}$ ) who have observed the influence of electric-field induced dipole alignment on the ESR spectrum of the hole centre in smoky quartz, which has dipolar properties.

In this paper we shall describe an experiment in which we observed a modulation of the optical-absorption coefficient of smoky quartz by means of an external electric field. In the first instance one would expect such an effect to be present since both the optical absorption in the visible region and the specific dielectric properties of X-irradiated smoky quartz are presumably due to the same hole centre. Electro-modulated absorption, due to dipole alignment, was described previously for an ionic dipole centre $\left(\mathrm{OH}^{-}\right.$in $\left.\mathrm{KCl}\right)$ by Kuhn and Lüty $\left.{ }^{8}\right)$. As far as we know such an electro-optic effect has not been reported previously for an electronic dipole centre*9).

2. Some remarks on the colour centre in smoky quartz. The lattice type of $\alpha$-quartz is trigonal; the point group, $\mathrm{D}_{3}$, has a threefold screw axis (the optical or $c$ axis) and three twofold axes ( $a$ axes) perpendicular to it. The unit cell contains three molecules $\mathrm{SiO}_{2}$; each silicon lies on an $a$ axis (see fig. 1a) and is surrounded by a tetrahedron of oxygens (fig. 1b). This tetrahedron is slightly distorted; the two $\mathrm{SiO}$ bond distances making an

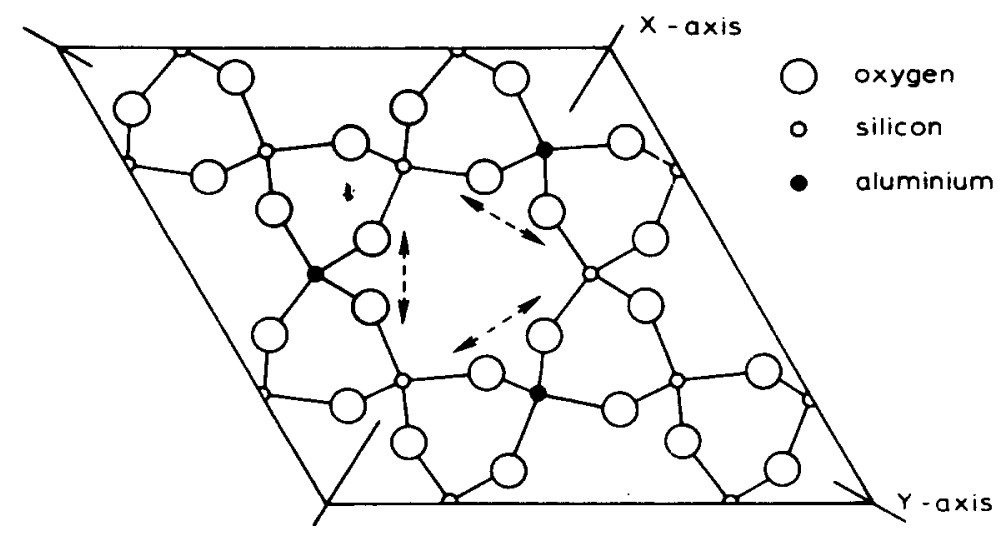

Fig. 1a. Projection of the ions in quartz on the plane perpendicular to the $c$ axis, showing the three possible $\mathrm{Al}^{3+}$ positions and the three oxygen pairs between which the hole is assumed to move. The $X$ axis corresponds to a twofold $a$ axis. After ref. 7 .

*Effects in which bound small polarons in $\mathrm{NiO}: \mathrm{Li}$ are involved, resembling those described in this paper in several aspects, have been reported in ref. 9 recently. 


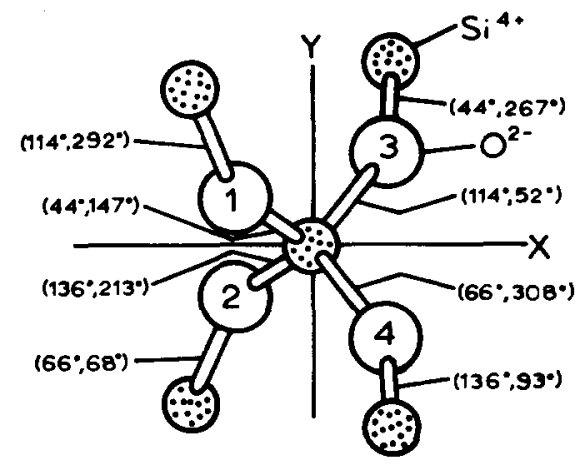

Fig. 1 b. The $\mathrm{SiO}_{4}$ tetrahedron in quartz; the $c$ axis and the $a$ axis correspond to the $Z$ axis, perpendicular to the plane of paper, and to the $X$ axis, respectively. The silicons and oxygens are denoted by $O$ and $O$. The numbers between parentheses denote the angles between the $\mathrm{SiO}$ bonds and the $Z$ axis and between their projections on the $X Y$ plane and the $X$ axis. After ref. 28.

angle of $66^{\circ}$ with the $c$ axis are somewhat longer than the two bond distances at an angle of $44^{\circ}$ with the $c$ axis. Every oxygen ion is shared by two tetrahedra.

In smoky quartz part of the silicon ions have been replaced by trivalent aluminium ions, accompanied by charge compensating monovalent alkali ions, say, sodium ions, in interstitial positions. By X-irradiation an electron may be released from one of the oxygen ions of an $\mathrm{AlO}_{4}$ complex and trapped at a $\mathrm{Na}^{+}$ion (at room temperature the $\mathrm{Na}$ atom diffuses away from the $\mathrm{Al}^{3+}$ ion), whereas a hole remains trapped at one of the oxygen ligands. The structure of this hole centre has been studied extensively by several authors $5,7,10-12$ ) by use of ESR techniques. The first theoretical analysis was presented by $\mathrm{O}^{\prime} \mathrm{Brien}^{13}$ ). The general outcome of these studies is first that, if the $\mathrm{Na}$ atom has been diffused away, the hole is trapped at the oxygens labelled $O(1)$ and $O(2)$ (fig. 1b). Secondly, the hole stays mainly in a nonbonding oxygen $\mathrm{p}$ orbital, labelled $\mathrm{p}_{x}$ and directed perpendicularly to the $\mathrm{Al}-\mathrm{O}-\mathrm{Si}$ plane. (In addition, the wave function of the hole slightly overlaps onto the aluminium nucleus, giving rise to hyperfine interaction.)

The optical absorption spectrum in the visible region ${ }^{12,14-16}$ ) exhibits a broad band around $450 \mathrm{~nm}$ and a much weaker one around $610 \mathrm{~nm}$. Both bands are usually attributed to excitations of the hole. In the O'Brien model the main absorption peak corresponds to a transition from the $\mathrm{p}_{x}$ orbital to a bonding sp orbital. The colour centre and its related ESR spectrum may be removed by heating to 250 to $350^{\circ} \mathrm{C}$, or optically by u.v. light. 


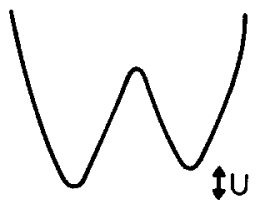

Fig. 2. One-dimensional asymmetric double-well potential in which the hole (see text) is assumed to move via a tunneling or jumping mechanism.

Because of the twofold site degeneracy the hole may be considered to move in a double-well potential. As the tunnel matrix element turns out to be very small (about $5 \times 10^{-7} \mathrm{eV}$ ) ${ }^{17}$ ), internal fields due to imperfections etc. are very important, resulting in an asymmetric form of the potential (see fig. 2; values of $U$ up to about $1.5 \mathrm{meV}$ were observed) ${ }^{17}$ ). Therefore, the hole will be highly localized at low temperatures; at high temperatures, in particular above $150 \mathrm{~K}$, the hole becomes increasingly delocalized due to the thermally activated hopping over the potential barrier, resulting in a disappearance ${ }^{18}$ ) of the ESR spectrum. The tunneling or hopping motion of the hole is equivalent to the reorientation of a dipole, giving rise to dielectric relaxation losses ${ }^{19}$ ), which have been studied extensively by De Vos and Volger ${ }^{17}$ ). These authors, like Taylor and $\mathrm{Farnell}^{7}$ ), could observe in their experiments the localization of the hole by applying large static electric fields.

3. Experimental procedures. The high voltage, applied to the specimen, was chopped by a reed relais, driven by a square-wave generator. The operating frequency was $60 \mathrm{~Hz}$. Light from a halogen or deuterium lamp passed through a monochromator, a linear polarizer, and subsequently through the crystal. The transmitted signal was detected by a photomultiplier (RCA, 1P28 or 1P22). The a.c. component of the output of the photomultiplier was examined with a lock-in detector which was synchronized by the square-wave generator. The d.c. component was also recorded. By comparing both components we could determine the electric-field induced change $\boldsymbol{\Delta}$ in the absorption coefficient $\boldsymbol{\alpha}_{t}$

The experiments were carried out below room temperature with the samples mounted in a helium cryostat. The temperature was measured with a germanium or copper resistor; the lowest temperature attainable was about $8 \mathrm{~K}$. If necessary, the, high voltage could be interrupted by means of a mechanical switch, located close to the sample.

The samples investigated were plane parallel platelets, cut from boules of natural smoky quartz and oriented perpendicular to either the $c$ axis or an $a$ axis. After polishing with diamond abrasive, semitransparent-nickel 
electrodes were applied by means of vacuum deposition; these electrodes show a rather flat transmission in the visible region and are of a reasonable mechanical strength. In our experiments we employed a configuration in which the propagation direction of the light coincided with the direction of the applied field. Most of the specimens were about $0.2 \mathrm{~mm}$ thick; the corresponding maximum value of the electric field we used was about $75 \mathrm{kV} / \mathrm{cm}$.

Usually, the crystal was thermally bleached by heating to about $400^{\circ} \mathrm{C}$ and subsequently recoloured by $\mathrm{X}$-irradiation for some time. The irradiation was performed at room temperature by means of a $50 \mathrm{kV}$, $32 \mathrm{~mA}$ tube with a tungsten anode, supplying a flux of $0.3 \mathrm{Mrad} / \mathrm{h}$.

The optical absorption spectrum was registrated for a few samples at room temperature with the help of a double-beam spectrometer (Perkin and Elmer EPS-3T). In addition, the dielectric loss of the samples was measured on a Schering bridge (GR $716 \mathrm{C}$ ).

4. Experimental results. It appears from our experiments that the effects in general are considerably larger for samples with the $a$ axis perpendicular than for those with the $c$ axis perpendicular to the main dimension of the sample. We shall call these orientations the $a$ and $c$ orientation, respectively, and shall treat the two cases separately since in some aspects the effects are different for the two orientations.

4.1. Measurements in the $a$ orientation. We shall restrict ourselves mainly to the experiments performed on the sample O-11 (X-irradiated for six hours), which is the most extensively investigated one on account of the magnitude of the effects for this specimen. The results, however, are also representative for the other samples investigated.

In fig. 3 we plotted the change $\Delta$ in the absorption coefficient, induced by an electric field $F=50 \mathrm{kV} / \mathrm{cm}$, at a temperature $T$ of $8.4 \mathrm{~K}$, as a function of the photon energy $\varepsilon$. The result is characteristic: $\Delta$ exhibits a maximum at $2.32 \pm 0.02 \mathrm{eV}(535 \mathrm{~nm})$ with a width at half-maximum of $0.83 \pm 0.03 \mathrm{eV}$, independent of the specific sample, the temperature and the irradiation time. The spectral dependence of $\Delta$ at $80 \mathrm{~K}$ does not differ from the one at $8 \mathrm{~K}$ but at room temperature a secondary maximum (with opposite sign with respect to the peak at $2.32 \mathrm{eV}$ ) appears around $3.2 \mathrm{eV}$ (fig. 4).

$\Delta$ depends linearly on the field, up to about $70 \mathrm{kV} / \mathrm{cm}$, except at very low temperatures, where above $60 \mathrm{kV} / \mathrm{cm}$ a tendency to saturation becomes noticeable (fig. 5). (After having performed other experiments with this sample, we ground it to a thickness of about $0.1 \mathrm{~mm}$, and applied a maximum field of about $140 \mathrm{kV} / \mathrm{cm}$. Above $110 \mathrm{kV} / \mathrm{cm}$ we 
could indeed observe a saturation, at least at $8.4 \mathrm{~K}$; see fig. 15.) The sign of $\Delta$ changes when the direction of the field is reversed.

Fig. 6 shows the way in which $\Delta$ depends on the azimuth $\phi$ of the polarizer (at $\phi=0$ the light is polarized parallel to the $c$ axis). This diagram is characteristic for all photon energies: $\Delta$ behaves according to $\sin ^{2} \phi$. In the experiments presented in fig. 3 , etc., $\phi$ was chosen in such a way that $\Delta$ was maximum.

Below $20 \mathrm{~K}$ the effect is almost constant with respect to $T$; above this value $\Delta$ decreases with rising temperature according to a $T^{-n}$ law (see fig. 7). For $n$ we found values between 1.2 and 1.6, varying from sample to sample.

4.2. Measurements in the $c$ orientation. Specimens in the $c$ orientation behave in a similar way as the $a$ oriented samples as far as the spectral, the field and the temperature dependence of $\Delta$ are concerned; the magnitude of the effect, however, is much smaller. The way in which $\Delta$ depends on the polarization angle of the light differs markedly for the two orientations.

All the investigated $c$-oriented samples, except one, exhibited a polarization diagram according to $\sin 2 \phi$. A typical example has been given in fig. 8 for sample L-2. We have also included in this figure the experimental points belonging to the reversed field direction because, for this specific sample, the absolute magnitude of $\Delta$ is not exactly the same for both field directions. This difference is absent at liquid-nitrogen temperatures.

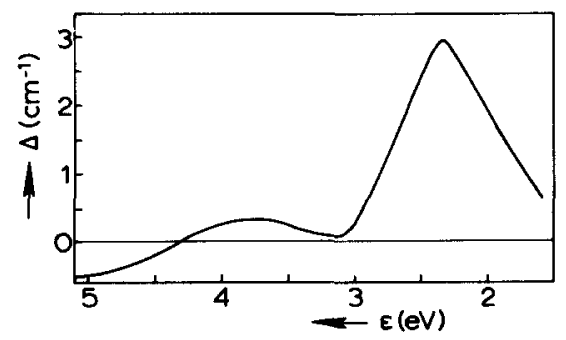

Fig. 3.

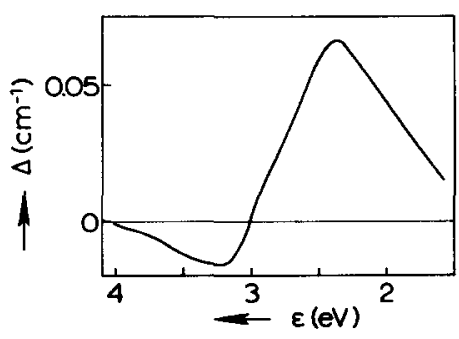

Fig. 4.

Fig. 3. The dependence of $\Delta$ on the photon energy $\varepsilon$ for sample $0-11$, measured at $F=$ $50 \mathrm{kV} / \mathrm{cm}$ and $T=8.3 \mathrm{~K}$.

Fig. 4. The same as in fig. 3 , but now measured at room temperature. 


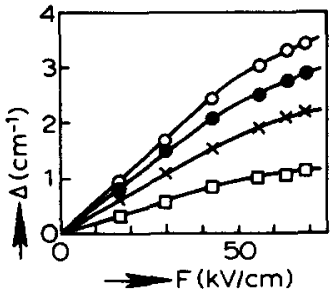

Fig. 5.

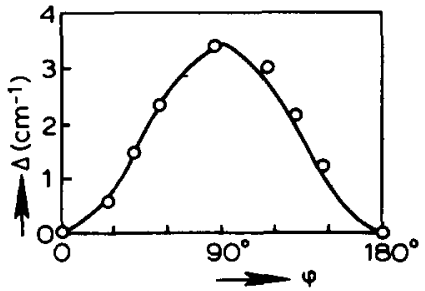

Fig. 6.

Fig. 5. The field dependence of $\Delta$ measured at $\varepsilon=2.27(0), 2.52(\bullet), 1.92(X)$ and $2.80(\square) \mathrm{eV} .(0-11 ; T=8.4 \mathrm{~K})$.

Fig. 6. Typical polarization diagram for the $a$ orientation $(\mathrm{O}-11 ; F=68 \mathrm{kV} / \mathrm{cm}, T=$ $8.3 \mathrm{~K}, \varepsilon=2.27 \mathrm{eV}$ ); the drawn curve is a $\sin ^{2} \phi$ function.

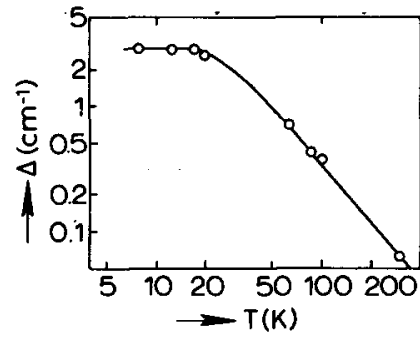

Fig. 7.

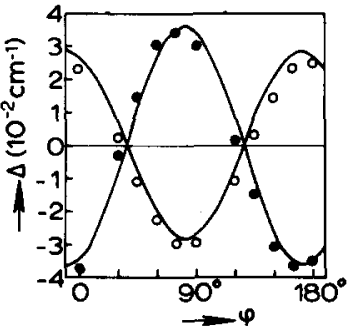

Fig. 8.

Fig. 7. Plot of $\Delta$ versus $T$; above $20 \mathrm{~K} \Delta$ behaves like $T^{-n}$ where $n>1(0-11 ; F=50$ $\mathrm{kV} / \mathrm{cm}, \varepsilon=2.27 \mathrm{eV}$ ).

Fig. 8. Typical polarization diagrams for the $c$ orientation for both signs of the field ( $O$ and $\bullet)$. The drawn curves are $\sin (2 \phi+\chi)$ functions. The difference between the magnitude for both cases is specific for the specimen $\mathrm{L}-2(F=50 \mathrm{kV} / \mathrm{cm}, \varepsilon=2.27 \mathrm{eV}$, $T=8.1 \mathrm{~K}$ ).

We found a strongly deviating behaviour for sample $0-2$, exhibiting a polarization dependence according to $A_{1}-A_{2} \sin 2 \phi$, i.e., an additional term independent of the polarization angle. The way in which $A_{1}$ and $A_{2}$ vary with $\varepsilon$ has been shown in fig. 9. Another remarkable particularity of this specimen is that $\Delta$ does not change its sign when the field direction is reversed.

4.3. The influence of the $X$-irradiation. We investigated how $\Delta$ depends on the X-irradiation time $t$. The experiments were performed on sample $0-11$ ( $a$ orientation). In fig. 10 we have reproduced the relation 
between $\Delta$ and $\varepsilon$ at $83 \mathrm{~K}$ for several irradiation times. From these results it may be inferred that neither the position of the main peak nor its width vary significantly with $t$. The height of the peak increases with $t$ according to $\left[1-\exp \left(-t / t_{0}\right)\right]$ where $t_{0} \simeq 1$ hour.

In addition, we recorded the absorption spectra of the irradiated crystal (with the light polarized parallel or perpendicular to the $c$ axis). An example has been given in fig. 11. The result is in agreement with that reported previously by other authors ${ }^{12,14-16}$ ). The absorption coefficient $\alpha$ grows in a similar way as $\Delta$ with a characteristic time of the same order of magnitude.

Besides an influence on the magnitude of $\boldsymbol{\Delta}$ we also found a small influence on the temperature dependence of the effect. We observed an increase in the value of the exponent $n$ (see section 4.1) from $n \simeq 1.40$ at $t=20 \mathrm{~min}$ up to $n \simeq 1.56$ at $t=6 \mathrm{~h}$.

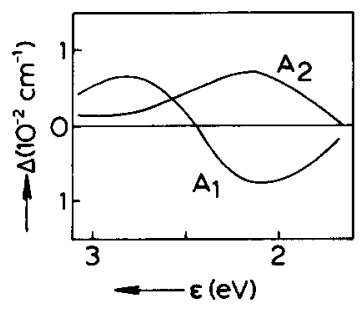

Fig. 9.

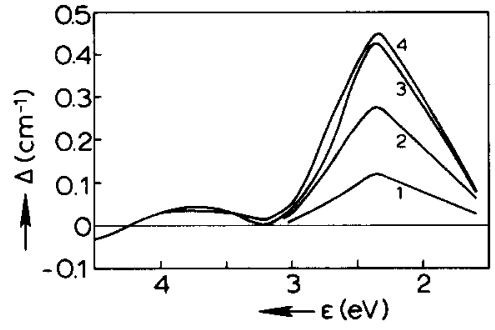

Fig. 10.

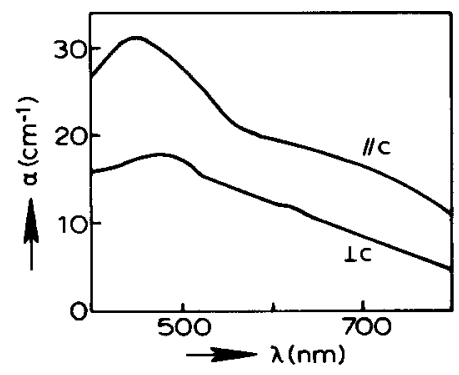

Fig. 11

Fig. 9. Spectral dependence of the constants $A_{1}$ and $A_{2}$ in the expression $\Delta_{1}=A_{1}-A_{n} \sin 2 \phi$ which holds for sample $0-2(T=8.7 \mathrm{~K} . F=64 \mathrm{kV} / \mathrm{cm})$.

Fig. 10. Spectral dependence of $\Delta$, measured after a total X-irradiation time $t=0.3,1$, 3 and $6 \mathrm{~h}$ (curves 1 to 4$)(0-11 ; F=50 \mathrm{kV} / \mathrm{cm}, T=83 \mathrm{~K})$.

Fig. 11. The absorption coefficient a versus the wavelength $\lambda$, measured for light polarized parallel to or perpendicular to the $c$ axis $(0-11 ; 6 \mathrm{~h} \mathrm{X}$-irradiated). 
4.4. Comparison between the investigated samples. In tables I and II the values of $\boldsymbol{\Delta}$ for the investigated samples, as measured in the liquidhelium region at $F=50 \mathrm{kV} / \mathrm{cm}$ and $\varepsilon=2.27 \mathrm{eV}$, have been collected for the $a$ and $c$ orientation, respectively. If the above-described experimental facts are connected with the presence of the aluminium colour centres we have to know the concentration of these centres in order to make possible a comparison between the crystals. For that reason we measured the dielectric relaxation loss of the crystals (at $0.3 \mathrm{MHz}$, giving rise to a Debye loss curve around $90 \mathrm{~K}$ ) since generally the maximum value of $T \tan \delta$ (where $\delta$ is the loss angle) is proportional to the dipole concentration $N$ :

$$
\left[T \tan \delta_{\max }\right]_{i}=\left(2 \pi / \varepsilon_{\mathrm{s}}\right)\left(p_{i}^{2} / k\right) N
$$

in which $\varepsilon_{\mathrm{s}}$ denotes the static dielectric constant, $k$ Boltzmann's constant and $p_{i}$ the average component of the dipole moment along the electric field, applied parallel to the $a$ or $c$ axes. De Vos and Volger ${ }^{17}$ ) have found for $p_{c}$ a value of $8.5 \pm 1.5$ debye (including local field corrections) and for the ratio between the components: $p_{c}^{2} / p_{a}^{2} \simeq 2$.

We have also included in the tables the experimental values of $T \tan \delta_{\max }$ and the derived values of the ratio $\Delta / N$.

TABLE I

\begin{tabular}{lccc}
\hline \multicolumn{4}{c}{ Some results for the $a$ orientation } \\
\hline Sample & $0-20$ & $0-11$ & $\mathrm{~L}-5$ \\
\hline$\Delta\left(\mathrm{cm}^{-1}\right)$ & 1.05 & 2.93 & 0.7 \\
$T \tan \delta_{\max }(\mathrm{K})$ & 0.36 & 0.34 & 0.21 \\
$\Delta / N\left(10^{-20} \mathrm{~cm}^{+2}\right)$ & 97 & 287 & 111 \\
\hline
\end{tabular}

TABLE II

\begin{tabular}{lccccc}
\hline \multicolumn{7}{c}{ Some results for the $c$ orientation } \\
\hline \multicolumn{1}{c}{ Sample } & $0-2$ & $\mathrm{~L}-2$ & $\mathrm{~L}-4$ & $\mathrm{~K}-5$ & $\mathrm{~S}-12$ \\
\hline$\Delta\left(10^{-2} \mathrm{~cm}^{-1}\right)$ & 1.3 & 3.6 & 2.9 & 0.3 & $<0.1$ \\
$T \tan \delta_{\max }(\mathrm{K})$ & 0.34 & 0.15 & 0.19 & 0.29 & 0.30 \\
$\Delta / N\left(10^{-20} \mathrm{~cm}^{+2}\right)$ & 2.5 & 16 & 10 & 0.7 & $<0.2$ \\
\hline
\end{tabular}


Both the absorption coefficient and the loss $T \tan \delta$ are proportional to the colour-centre concentration $N$. Indeed we found the absorption coefficients of $0-11$ and $0-20$ to be almost the same (about $30 \mathrm{~cm}^{-1}$ at $450 \mathrm{~nm}$ for light polarized parallel to the $c$ axis).

5. Some features of a model of anisotropic dipolar colour centres. We want to discuss our experimental results in terms of a model which we will present in this section. Starting point will be the assumption that we are dealing with a colour centre exhibiting anisotropic optical absorption. More specifically, we will assume the following*. (i) The absorption coefficient $\alpha$ has axial symmetry and may be written as

$$
\alpha(\tau)=\alpha / / \cos ^{2} \tau+\alpha_{\perp} \sin ^{2} \tau=\alpha_{\perp}+\Delta \alpha \cos ^{2} \tau
$$

where $\Delta \alpha=\alpha / /-\alpha_{\perp}$ and $\tau$ denotes the angle between the polarization direction of the light and the symmetry axis of the centre, which we shall call the optical axis $L$ of the centre. (ii) The centre behaves like an electric dipole which may occupy two different positions $p_{1}$ and $p_{2}$. As usually such a situation may be described by ${ }^{19}$ ) a double-well potential as depicted in fig. 2 , the energy difference

$$
E_{1}-E_{2}=U
$$

between the two positions being due to local fields, strains etc. (iii) The optical axis changes with the dipole axis: reorientation of the dipole from $p_{1}$ to $p_{2}$ is accompanied by reorientation of $L$ from $L_{1}$ to $L_{2}$.

5.1. Temperature and field dependence. The absorption coefficient of the centre may be written quite generally as an average over the two positions, so that

$$
\alpha(T)=\left[\alpha\left(\tau_{1}\right) \exp \left(-E_{1} / k T\right)+\alpha\left(\tau_{2}\right) \exp \left(-E_{2} / k T\right)\right] Z^{-1},
$$

in which $Z=\exp \left(-E_{1} / k T\right)+\exp \left(-E_{2} / k T\right)$ denotes the partition function. Inserting eq. (2) into eq. (3) leads to

$$
\alpha(T)=\alpha_{\perp}+\Delta \alpha \frac{\cos ^{2} \tau_{1}+\cos ^{2} \tau_{2} \exp (U / k T)}{1+\exp (U / k T)} .
$$

*Most of the assumptions do not differ essentially from those in ref. 8 . 
Besides a temperature dependence for $\alpha$ eq. (4) implies that the absorption may be altered upon changing $U$. This may be accomplished by an externally applied electric field $F$. Then, eq. (2) should be replaced by

$$
E_{1}-E_{2}=U+p_{\circ} \cdot F,
$$

where

$$
p_{\mathrm{o}}=p_{2}-p_{1} .
$$

(Internal field corrections are assumed to be included in the dipole moments.) Henceforth we will label the orientation in which the angle between $F$ and the dipole is the largest, by the index 1. (Consequently, $p_{\mathrm{o}} \cdot \boldsymbol{F}$ does not depend on the sign of $\boldsymbol{F}$.)

In the presence of the external field eq. (4) then takes on the form

$$
\alpha(F)=\alpha_{\perp}+\Delta \alpha \frac{\cos ^{2} \tau_{1}+\cos ^{2} \tau_{2} \exp \left(U+p_{0} \cdot F\right) / k T}{1+\exp \left(\left(U+p_{0} \cdot F\right) / k T\right)} .
$$

Making the reasonable assumption that in the real crystal the electricdipole system does not show a zero-field polarization, i.e. the number of centres with $E_{1}-E_{2}=U$ equals the number with $E_{1}-E_{2}=-U$, we can derive from eq. (6) for the quantity

$$
\Delta(F)=\alpha(F)-\alpha(0),
$$

the following expression:

$$
\Delta(F)=\Delta_{\mathrm{s}} \kappa(F),
$$

in which

$$
\Delta_{\mathrm{S}}=\frac{1}{2}\left(\cos ^{2} \tau_{2}-\cos ^{2} \tau_{1}\right) \Delta \alpha
$$

and

$$
\boldsymbol{\kappa}(F)=\frac{\sinh \left(\boldsymbol{p}_{\mathrm{o}} \cdot \boldsymbol{F} / k T\right)}{\cosh \left(\boldsymbol{p}_{\mathrm{o}} \cdot \boldsymbol{F} / k T\right)+\cosh (U / k T)} .
$$

Since the sign of $\Delta_{\mathrm{s}}$ changes when the field is reverse, in contrast to $\kappa(F)$, the sign of $\Delta(F)$ depends on the field direction. $\Delta(F)$ saturates at large fields: $\Delta(\infty)=\Delta$ as $\boldsymbol{x}(\infty)=1$, i.e. the dipole system is completely polarized. At high temperatures and small fields $\Delta$ depends linearly on $T^{-1}$ and $F$, respectively. 
Eq. (7) may be generalized straightforwardly by introducing a normalized distribution function $f(U)$ for the possible $U$ values,

$$
\int_{0}^{\infty} f(U) \mathrm{d} U=1
$$

Eq. (7) is then replaced by

$$
<\Delta(F)>=\int_{0}^{\infty} \Delta(F) f(U) \mathrm{d} U .
$$

In the case of a gaussian distribution with its maximum value at $U=0$ and a width $U_{0}$ eq. (8) gives

$$
<\Delta(F)>=\left(2 / \pi^{\frac{1}{2}}\right)\left(\Delta_{\mathrm{s}} / U_{\mathrm{o}}\right) \int_{0}^{\infty} \kappa(F) \exp \left[-\left(U / U_{\mathrm{o}}\right)^{2}\right] \mathrm{d} U
$$

In figs. $12 \mathrm{a}$ and $13 \mathrm{a}$ we plotted the dependence of $\kappa$, a function of $p F / k T$, on $F$ and $T^{-1}$, respectively, for several values of the parameter $U$. The corresponding results, after averaging $\kappa$ over a gaussian distribution according to (8a) with numerical methods, are presented in figs. $12 \mathrm{~b}$ and $13 \mathrm{~b}$ for several values of $U_{0}$. Qualitatively, $\kappa$ and $\langle\kappa\rangle$ appear to behave in a similar way with one exception: the effect that $\kappa$ disappears at low temperatures when the field is sufficiently low, i.e. when $U>p_{0} \cdot \boldsymbol{F}$ (see fig. 13a), is absent after the gaussian averaging.

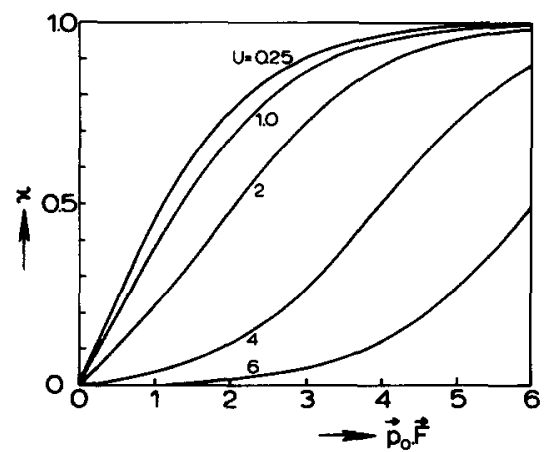

Fig. 12a.

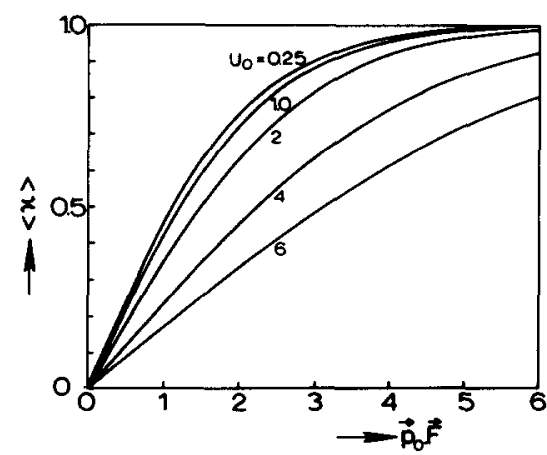

Fig. $12 b$.

Fig. 12a. Field dependence of $\boldsymbol{x}$ for several values of the parameter $U ; p_{0} \cdot \boldsymbol{F}$ and $U$ in units of $k T$.

Fig. $12 \mathrm{~b}$. Field dependence of $\langle\boldsymbol{\kappa}\rangle$ for several values of $U_{\mathrm{o}}$ [see eq. (8a)]; $\boldsymbol{p}_{\mathrm{o}} \cdot \boldsymbol{F}$ and $U_{\mathrm{o}}$ in units of $k T$. 


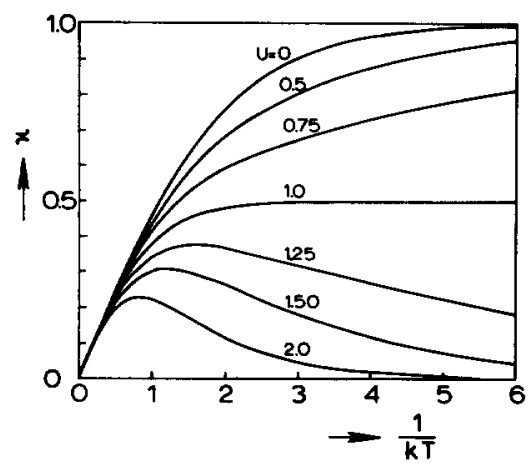

Fig. 13a.

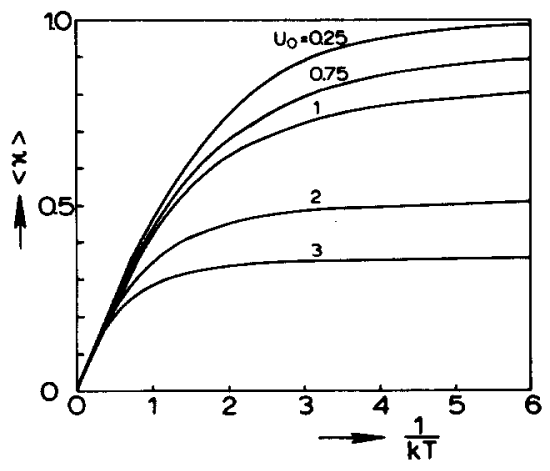

Fig. 13b.

Fig. 13a. Temperature dependence of $\boldsymbol{\kappa}$ for several values of $U ; U$ and $k T$ in units of $p_{0} \cdot F$.

Fig. 13b. Temperature dependence of $\langle\kappa\rangle$ for several values of $U_{\mathrm{o}}$ [eq. (8a)]; $U_{\mathrm{o}}$ and $k T$ in units of $p_{0} \cdot F$.

5.2. Polarization dependence. In order to specify the orientations $p_{1}$ and $p_{2}$ of the dipole we choose a coordinate system in such a way that the projections of $p_{1}$ and $p_{2}$ on the $z$ azis are equal in magnitude but with opposite signs. For convenience we suppose $L$ to coincide with the dipole direction. We specify the orientation of the dipole by the angle $\eta$ between $p$ and the $z$ axis and the angle $\theta$, between the projection on the $x-y$ plane and the $y$ axis (see fig. 14a.).

First we consider the case of the electric field $F$ applied along the $y$ axis, parallel to the propagation direction of the light. The angle between the electric vector $E$ of the incident linearly polarized radiation and the $z$ axis will be denoted by $\phi$. For this situation one finds for the quantity $\cos ^{2} \tau$ which is involved in expression (7a) for $\Delta_{s}$ :

$$
\cos ^{2} \tau=\sin ^{2} \eta \sin ^{2} \theta \sin ^{2} \phi+\cos ^{2} \eta \cos ^{2} \phi+\frac{1}{2} \sin 2 \eta \sin \theta \sin 2 \phi .
$$

Consequently we have (calling $\boldsymbol{\eta}_{1} \boldsymbol{\eta}$ and using $\boldsymbol{\eta}_{2}=\boldsymbol{\pi}-\boldsymbol{\eta}_{1}$ ):

$$
\begin{aligned}
p_{\mathrm{o}} \cdot F=p F \sin \eta\left(\cos \theta_{1}-\cos \theta_{2}\right), \\
\begin{aligned}
\cos ^{2} \tau_{2}-\cos ^{2} \tau_{1}= & \sin ^{2} \eta\left(\sin ^{2} \theta_{2}-\sin ^{2} \theta_{1}\right) \sin ^{2} \phi \\
& -\frac{1}{2} \sin 2 \eta\left(\sin \theta_{1}+\sin \theta_{2}\right) \sin 2 \phi .
\end{aligned}
\end{aligned}
$$

The right-hand side of (10) may be abbreviated

$$
g(\phi)=a \sin ^{2} \phi-b \sin 2 \phi .
$$




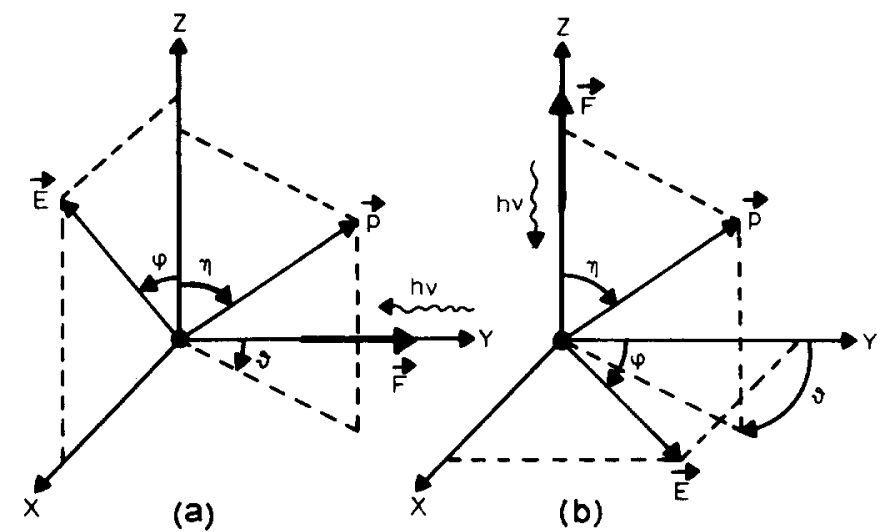

Fig. 14. The coordinate system, defining the orientation of $\boldsymbol{p}$ and $\boldsymbol{E}$ for the applied field and the incident light perpendicular to (a) or parallel to (b) the $z$ axis.

This function has zeros at values of $\phi$ given by $\tan \phi=2 b / a$ and at $\phi=0$ and $\pi$ and extrema for $\phi$ given by $\tan 2 \phi=2 b / a$. Therefore $\Delta_{\mathrm{s}}$. will in general depend on the polarization angle in a complicated way unless $a=0$ or $b=0$ in (10a).

We shall now extend this description by requiring the $z$ axis to be a trigonal axis of the crystal, accompanied by the presence of three equivalent dipoles instead of one. (Obviously this situation refers to the case of quartz, the $z$ axis being the optical $c$ axis and, according to our experimental configuration (section 4), the $y$ axis being the $a$ axis). As a consequence we now have to average eqs. (10) or (10a) over the three dipoles. Since in the case of trigonal symmetry $\Sigma_{i}\left(\sin \theta_{1}+\sin \theta_{2}\right)_{i}=0$ we find that the average value behaves like

$$
\left[\cos ^{2} \tau_{2}-\cos ^{2} \tau_{1}\right]_{\mathrm{av}}=A \sin ^{2} \phi .
$$

Secondly we want to consider the situation (fig. 14b) where the electric field and the propagation direction of the light are parallel to the trigonal $z$ axis (this case refers to the experimental $c$ orientation: section 4). For a single dipole one finds

$$
\boldsymbol{p}_{\mathrm{o}} \cdot \boldsymbol{F}=2 p F \cos \boldsymbol{\eta}
$$

and

$$
\cos ^{2} \tau_{2}-\cos ^{2} \tau_{1}=\sin ^{2} \eta\left[\cos ^{2}\left(\phi-\theta_{2}\right)-\cos ^{2}\left(\phi-\theta_{1}\right)\right],
$$

the latter having a dependence like

$$
\cos ^{2} \tau_{2}-\cos ^{2} \tau_{1}=A \sin (2 \phi+\chi) \text {. }
$$


Taking into account the trigonal symmetry the averaging procedure yields

$$
\left[\cos ^{2} \tau_{2}-\cos ^{2} \tau_{1}\right]_{\mathrm{av}}=0
$$

and $\Delta(F)$ therefore vanishes.

Finally we calculate the absorption coefficient itself (without field) by averaging eq. (1) over the possible positions and orientations:

$$
\alpha_{\mathrm{av}}=\frac{1}{3} \sum_{i}\left[\alpha_{1}+\frac{1}{2} \cdot \Delta \alpha\left(\cos ^{2} \tau_{1}+\cos ^{2} \tau_{2}\right)_{i}\right]
$$

For the special cases of light polarized parallel to the $z$ axis $(\phi=0)$ or perpendicular to it $\left(\phi=\frac{1}{2} \pi\right)$ one gets

$$
\alpha_{\mathrm{av}}(0)=\alpha_{\perp}+\Delta \alpha \cos ^{2} \eta \text { and } \alpha_{\mathrm{av}}\left(\frac{1}{2} \pi\right)=\alpha_{\perp}+\frac{1}{2} \Delta \alpha \sin ^{2} \eta
$$

and for the dichroism

$$
\Pi=\frac{\alpha_{\mathrm{av}}(0)}{\alpha_{\mathrm{av}}\left(\frac{1}{2} \pi\right)}=\frac{\alpha_{\perp}+\Delta \alpha \cos ^{2} \eta}{\alpha_{\perp}+\frac{1}{2} \Delta \alpha \sin ^{2} \eta} .
$$

By combining (16) and (7a) the spectral dependence of $\Delta_{\mathrm{S}}$ can be deduced from the spectral dependences of $\alpha_{a v}(0)$ and $\alpha_{a v}\left(\frac{1}{2} \pi\right)$ to be

$$
\Delta_{\mathrm{s}}(:) \boldsymbol{\alpha}_{\mathrm{av}}(0)-\alpha_{\mathrm{av}}\left(\frac{1}{2} \pi\right)
$$

inplying that in the limiting case of $\alpha_{1}=0$ (or $\alpha_{/ /}=0$ ) $\Delta_{\mathrm{s}}$ closely follows the absorption coefficient itself.

6. Discussion. 6.1. Attempt to attribute the results to the Al centre. First we shall try to relate the experimental results concerning the electro-modulated absorption to the presence of the aluminium colour centre and to describe these in terms of the model analyzed above. We then have to introduce the specific properties of the Al centre into the model. The dipole connected with the centre may point either in the $\mathrm{AlO}(1)$ or in the $\mathrm{AlO}(2)$ directions (fig. $1 \mathrm{~b}$ ), which gives rise to two dipole orien tations $p_{1}$ and $p_{2}$ making equal angles (in casu $44^{\circ}$ ) with the positive or negative $z(c)$ axis (i.e., $\eta_{2}=180^{\circ}-\eta_{1}$ ). This was already taken into account in the model as was the trigonal symmetry of quartz. The symmetry properties of the absorption coefficient are determined by the oscillator strengths of the transitions or, more explicitly, by matrix elements of the kind $\langle i|r| f\rangle$, where $|i\rangle$ and $|f\rangle$ are the initial and final wave functions. As already mentioned in section $2|i\rangle$ is taken as the $p_{x}$ oxygen orbital, while $|f\rangle$ has 
the character of an s orbital. Consequently, the optical axis $L$ of the centre coincides with the symmetry axis of the $\mathrm{p}_{x}$ orbital, i.e. is perpendicular to the $\mathrm{Si}-\mathrm{O}(1$ or 2$)-\mathrm{Al}$ plane, so that $\alpha_{\perp}=0$. The angle between the two possible $L$ directions and the positive or negative $z$ axis, respectively, may be calculated to be $51^{\circ}$ : their projections on the $x y$ plane coincide. (Note that in this case the direction of $L$ differs from $\boldsymbol{p}$.) This latter result means that in eqs. (10) and (13) [not in (9)] we must put $\theta_{1}$ $=\theta_{2}$. Therefore, we have to conclude that owing to this fact and to the trigonal symmetry all possible contributions to $\Delta_{\mathrm{av}}$ cancel, or, in other words, the aluminium centre cannot be held responsible for the electromodulation of the absorption.

Deviations from the undisturbed "ideal" orientations of $L$ may be considered. For instance $\eta_{2} \neq 180^{\circ}-\eta_{1}$ and/or $\theta_{1} \neq \theta_{2}$ for one or more positions of the centre in the unit cell. In some cases these deviations give rise to nonvanishing contributions to $\Delta_{\mathrm{av}}$, which can be distinguished because of their polarization dependences. This does not lead to reasonable results. Another cause for the occurrence of an effect could be an unequal population of the three possible aluminium sites in the unit cell. This phenomenon has been reported a few times in the literature ${ }^{10,12,20}$ ), but its occurrence seems to be rather incidental. The population difference would introduce a term like $B \sin 2 \varphi$ in the polarization dependence in the $a$ orientation, which was not observed.

If the hole would occupy an $\mathrm{O}(3)$ or $\mathrm{O}(4)$ position, the angle between the corresponding direction of $L$ and the positive or negative $z$ axis would turn out to be $67^{\circ}$ and the angle between the projection of $L$ on the $x y$ plane and the $a$ axis through the $\mathrm{Al}$ ion to be $\pm 27^{\circ}$, leading to $\theta_{1}-\theta_{2}=$ $54^{\circ}$. In this case the effects would certainly not disappear. However, the ground state of the hole in smoky quartz, irradiated at room temperature, is situated at the $\mathrm{O}(1)$ and $\mathrm{O}(2)$ positions. Since occupation of both of the other positions occurs via thermal excitation ${ }^{12}$ ), it is not possible to explain the occurrence of the effects below, say, $20 \mathrm{~K}$ in this way (see, however, section 6.4).

Irrespective of all these arguments, we should criticize an assignment of the observed modulation to the aluminium centre for two experimental reasons. First, contrary to what is to be expected if the Al centre should be involved, the data of tables I and II - do not reveal a relationship between the colour-centre concentration and the magnitude of $\Delta$. Secondly, the spectral dependences of $\Delta$ (fig. 3 ) are not related in any sense to the absorption coefficient ( $c f$. section 5.2).

6.2. Attribution of the results to a $\mathrm{XO}_{4}$ complex. Although the observed phenomena apparently cannot be attributed to the aluminium 
centre, they suggest in any case that they may be attributed to a colour centre with dipolar character. There is, indeed, at least a qualitative agreement between the observed field and temperature dependences of $\boldsymbol{\Delta}$ and the dependences as predicted for the model of section 5 by eqs. (7) or (8). $\Delta$ depends on the sign of the fields, increases linearly with the applied field, followed by saturation, and lowering of the temperature results in an increase of $\Delta$ up to saturation. Fig. 15 shows that the experimental values of $\boldsymbol{\kappa}$ as derived from the "saturation" experiment in section 4.1 may be fitted, at least roughly, by a theoretical curve $\langle\kappa\rangle$ as given by (7b) after a gaussian averaging according to (8) ( $c f$. fig. 12b). The adapted values of $U_{\mathrm{o}}$ and the projection of $p_{\mathrm{o}}$ on the $a$ axis $p_{\mathrm{o} a}$, are about 1.5 $\mathrm{meV}$ and 12 debye, respectively. They are just of the order of magnitude which one would expect ${ }^{17}$ ), if the dipolar character of the colour centre is determined by the hopping motion of a hole between two adjacent oxygen ions like in the aluminium centre.

Some extra information about the centre may be got from the polarization diagrams, if we assume that the centre has an optical axis $L$, as in section 5 , which points into one of two possible directions, depending on the direction of $\boldsymbol{p}_{0}$. If, for instance, the requirement $\eta_{2}=\pi-\eta_{1}$ is dropped in section 5.2 a term $c \cos ^{2} \phi$ arises in eq. (10a), or, in other words, $g(\phi)$ then contains a constant term which, moreover, does not vanish in general when the trigonal symmetry is introduced. Therefore, because such a behaviour was not observed, we infer, that the projections of $L_{1}$ and $L_{2}$ on the $x y$ plane are equal, as in the aluminium centre. Furthermore, the fact that the polarization behaves as $\sin ^{2} \phi$ [eq. (11)] and not as $g(\phi)$ [eq. (10a): confining ourselves to the $a$ orientation], indicates that also for this centre the $z$ axis is a trigonal symmetry axis of the crystal.

In view of these considerations we believe the colour centre which is held responsible for the electromodulation, to consist of an ion $\mathrm{X}$, for the moment unknown, substitutionally replacing $\mathrm{a} \mathrm{Si}^{4+}$ ion and accompanied

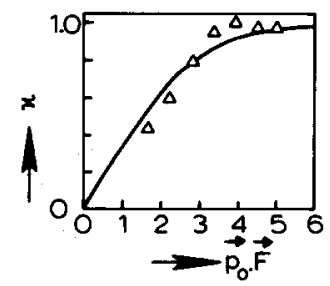

Fig. 15. Experimental values of $\kappa$, fitted by the theoretical expression eq. (8a) with $U_{\mathrm{o}}$ $=2 k T \simeq 17 \mathrm{~K}$ and $p_{\mathrm{oa}} \simeq 12$ debye $(0-11 ; \varepsilon=2.27 \mathrm{eV}, T=8.5 \mathrm{~K})$. 
by a hole, trapped at one of the surrounding oxygen ions, presumably at an $\mathrm{O}(1)$ or $\mathrm{O}(2)$ ion. From the dielectrical point of view the $\mathrm{XO}_{4}$ centre is completely analogous to the $\mathrm{AlO}_{4}$ centre. Its optical behaviour, however, must be quite different since it should depend strongly on the properties of the $\mathrm{X}$ ion. Moreover, realignment of the dipole involved by means of the electric field should give rise to a change in these properties. According to section 4.2 the relevant properties must be irradiation induced.

If this centre indeed behaves in full accordance with the model of section 5, we must explain the occurrence of the effects in the $c$ orientation [contrary to eq. (15)] by assuming that the $\mathrm{X}$ ions have a small preference for one specific lattice position. A population difference between the three available lattice positions removes the trigonal symmetry and leads to a $\Delta$ with a polarization dependence as given by (14) in agreement with our experimental results (fig. 8). The preference may indeed be assumed to be small since it was not observable in the experiments in the $a$ orientation. The fact that the observed values of $\Delta$ in the $c$ orientation are much smaller than those in the $a$ orientation (compare tables II and I, respectively) indicates, therefore, that we are dealing with a deviation of the "ideal" case $\Delta=0$ and not with accidentally large differences in concentrations between crystals in the $a$ and $c$ orientation.

The observed difference between $|\Delta(+F)|$ and $|\Delta(-F)|$ in the case of sample L-2 ( $c$ orientation, fig. 8 ) may be due to violation of one of the assumptions in the derivation of eq. (7), namely, that the number of centres with $E_{1}-E_{2}=U$ [eq. (2)] equals the number with $E_{1}-E_{2}=$ $-U$. The consequence of such a violation, which is perhaps caused by a specific internal strain field in this sample, can be quickly understood in a qualitative way, considering the extreme case in which no centres with $E_{1}-E_{2}=-U$ are present at all. With the help of eq. (6) one then easily finds

$$
|\Delta(+F) / \Delta(-F)|=\exp (-U / k T)
$$

expressing the fact that the difference between $|\Delta(+F)|$ and $|\Delta(-F)|$ is negligible at high temperatures but increases upon lowering of the temperature. Indeed, the effect was observed in the liquid-helium region but not at liquid-nitrogen temperature. The origin of the anomalous properties of the other sample which was found to exhibit an exceptional behaviour $(\mathrm{O}-2)$, is still enigmatic.

The agreement between the predicted and observed properties is much better for the field dependence and in particular for the polarization 
dependence than for the temperature dependence, as is revealed by a comparison between figs. $13 \mathrm{~b}$ and 7 . The observed saturation below $20 \mathrm{~K}$ is too pronounced and the decrease with rising $T$ too fast with respect to the theoretical behaviour $\left(T^{-1}\right)$. The main reason could be that in section 5 we neglected any temperature dependence of $\Delta \alpha$ to which $\Delta_{\mathrm{s}}$ is proportional [eq. (7a)]. From a comparison between figs. 13b and 7 one would conclude that $|\Delta \alpha|$ increases with $T$, arrives at a maximum at, say, $15 \mathrm{~K}$ and then decreases slowly. However, this cannot be the full explanation since we also observed a small influence of the X-irradiation on the temperature dependence (section 4.3). On account of its ionizing action the X-irradiation produces internal disturbing electric fields. If their strengths grow with the irradiation time and if a possible sensitivity of $\Delta \alpha$ for these fields is somewhat temperature dependent, both the influence of the irradiation and the differences between the samples in this respect may be understood.

\subsection{Proposal for the nature of the Xion. We now want to go into the} nature of the unknown ion $\mathrm{X}$ more explicitly. In our attempt to identify this ion we shall assume, as a starting point, that the spectral dependence of $\Delta$ in some way reflects the spectral behaviour of the absorption coefficient of the centre itself. We found a pronounced maximum in $\Delta$ at $535 \mathrm{~nm}$ (section 4.1). The only well-known centre in quartz which exhibits an absorption peak in this region, is the colour centre that is held responsible for the characteristic colour of amethyst ${ }^{21}$ ) (absorption around $545 \mathrm{~nm}$ ). Therefore, we tentatively propose to ascribe our experimental results in principle to this centre (although we shall have to modify it slightly later on).

In order to elucidate this assignment we first summarize some properties of the amethyst centre. On account of its ESR spectrum and its optical absorption, the centre was proposed by Moore and Lehmann ${ }^{22,23}$ ) to consist of a $\mathrm{Fe}^{4+}$ ion at a $\mathrm{Si}^{4+}$ site. The optical absorption is attributed to a charge-transfer transition of the type in which an electron is transferred from a bonding orbital of dominating ligand character to an unoccupied orbital, mostly localized on the central metal ion, in this case ${ }^{23}$ ) from a $t_{1} \pi$ orbital of the oxygens (p type) to the $e$ orbitals and $t_{2}$ orbitals of the iron $\left(\mathrm{d}_{z 2}\right.$ type and $\mathrm{d}_{x y}$ type, respectively). The $t_{1} \rightarrow e$ transition is then identified with the $545 \mathrm{~nm}$ band and the $t_{1} \rightarrow t_{2}$ and $e \rightarrow$ $t_{2}$ transitions with weaker bands around $350 \mathrm{~nm}$ and $950 \mathrm{~nm}$, respectively. The precursor ${ }^{24}$ ) of this irradiation-induced centre is a $\mathrm{Fe}^{3+}$ ion at a $\mathrm{Si}^{4+}$ site, accompanied by, say, a $\mathrm{Na}^{+}$ion. Under the influence of the $\mathrm{X}$-irradiation an electron may be released from this $\mathrm{Fe}^{3+}$ ion and captured by an interstitial ${ }^{25}$ ) $\mathrm{Fe}^{3+}$ ion. This suggests that the interstitial 
iron ion is a much deeper and more effective trap than the near sodium ion. It has been argued that the sodium remains in its original position. In general the behaviour of iron in quartz is quite complicated ${ }^{26}$ ).

We shall assume that the above model for the amethyst centre is correct, although it involves the tetravalent state of the iron ion which is rather unusual (its existence, however, has also been suggested in some other compounds $\left.{ }^{31}\right)$ ).

The identification of our $\mathrm{XO}_{4}$ centre with the amethyst centre requires the presence of iron in our samples; it is known, indeed, from chemical analysis that iron is a very common impurity in smoky quartz (as aluminium is in amethyst). We assume that the precursor of the amethyst centre is also present in smoky quartz. However, we suppose that under the influence of X-irradiation two electrons are released from it instead of one as in amethyst: one electron from the $\mathrm{Fe}^{3+}$ ion and one from an $\mathrm{O}^{2-}$ ion. One of them may be captured by the $\mathrm{Na}^{+}$ion which subsequently diffuses away, and the other by an electron trap $Y$ (which could be, for instance, a mono or divalent metal ion like $\mathrm{Na}^{+}$or $\mathrm{Ba}^{++}$, an interstitial iron ion, an oxygen vacancy ${ }^{27,28}$ ), or an $\mathrm{AlO}_{4}$ complex on which a hole is trapped). On account of the known behaviour of the Al centre (see section 2) and the results of the previous section, it is plausible that the hole trapped at the oxygens is localized mainly at one of them. Consequently the centre thus formed has the required dipolar character where the hopping motion of the hole between two adjacent oxygens embodies the reorientation of the dipole. In summary, in this hypothesis the iron centre is considered to be a mixing of the amethyst centre and the aluminium centre.

It should be pointed out that, if indeed the sodium ion has to be regarded as a real part of the amethyst centre, this centre is positively charged. Similarly the proposed iron centre in smoky quartz is charged, owing to the presence of the trapped hole. The presence of such centres, charged by trapped electrons or holes, in quartz is not unusual. However, they seem to require a sufficiently local charge compensation in order to be stable at room temperature or higher. The germanium centre ${ }^{10,28,29}$ ) in quartz, for instance, arising from a substitutional $\mathrm{Ge}^{4+}$ ion upon X-irradiation by trapping an electron (in an admix ture of a Ge s orbital and oxygen $\mathrm{p}$ orbitals) notwithstanding the fact that $\mathrm{Ge}^{4+}$ carries the same charge as $\mathrm{Si}^{4+}$, is stable at room temperature provided it is locally compensated by, e.g., an interstitial alkali ion. Considering this negatively charged centre to be a counterpart of the positively charged iron centre, it is suggested that some additional stabilization of the trapped hole can be achieved from the presence of a negatively charged defect. The above-mentioned electron trap $\mathrm{Y}$ which becomes negatively charged during the process of the 
colour-centre creation, can act in such a way if it is located in the surroundings of the centre.

On expects a priori that the three oxygens on which no hole is trapped play the most important part in the charge-transfer process. Therefore, it is evident that the optical and dielectric properties of the centre are coupled. As another possible mechanism for the desired coupling we propose the following. We suppose that the presence of the hole on one of the oxygens*), say, $\mathrm{O}(1)$, causes a small distortion of the $\mathrm{FeO}_{4}$ complex in such a way that the overlap between the $3 \mathrm{~d}$ orbitals of the iron and the $\pi$ orbitals of the other oxygen ions changes, say, increases with respect to the overlap in which the $O(1)$ ion is involved. Because the oscillator strength of the charge-transfer transition depends on the amount of overlap, the distortion introduces an optical anisotropy which is related directly to the position of the hole and consequently may be influenced by an external electric field.

In tables III and IV we visualized the mechanisms according to which the creation and optical absorption occur for the amethyst centre and the proposed model for the iron centre in smoky quartz, respectively.

6.4. Additional remarks. Some additional support for the idea that iron ions are involved may be obtained from the following facts. Contrary to the case of $\mathrm{Al}^{3+}$ ions in smoky quartz it is very common that the $\mathrm{Fe}^{3+}$ ions in amethyst are unequally distributed over the possible lattice positions ${ }^{21,24}$ ). This makes plausible our explanation for the occurrence of the effects in the $c$ orientation. The other fact is that a second chargetransfer transition takes place in the amethyst centre around $350 \mathrm{~nm}$. Fig. 3 shows that $\Delta$ also exhibits a secondary maximum in this region, namely around $335 \mathrm{~nm}(3.7 \mathrm{eV})$.

In our discussion of the aluminium centre we chose as a starting-point the O'Brien model. It should be remarked, however, that this model is still somewhat controversial as far as its optical aspects ${ }^{12,30}$ ) are concerned. The observed magnitude of the dichroism lends some support to the model. The experimental value of the dichroism at $450 \mathrm{~nm}$ varies between 1.5 and 1.8. From the average value $\Pi=1.65$ and using eq. (17) under the assumption $\alpha_{1}=0$ we may calculate the angle $\eta$, yielding a value of about $48^{\circ}$ in reasonable agreement with the theoretical value of $51^{\circ}$, which is based on the assumed correctness of the O'Brien model.

Our final remark concerns the (negative) peak in $\Delta$, observed around 3.2

*The presence of a hole at one of the oxygens reduces the symmetry of the $\mathrm{FeO}_{4}$ complex from $T_{d}$ to $C_{3 v}$, assuming the "undisturbed" complex to be a regular tetrahedron. 
TABLE III

Mechanisms for the creation and optical absorption of the iron colour centre in amethyst, as proposed in refs. 22 and 24

\begin{tabular}{|c|c|c|}
\hline Creation: & 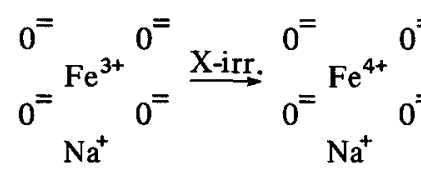 & $+\mathrm{e}\left(\rightarrow \mathrm{Fe}^{3+}\right.$ interstit \\
\hline Absorption: & $\begin{array}{l}0^{=}{ }^{0=}{ }_{\mathrm{Fe}^{4+}}{ }_{\mathrm{Na}^{+}}=\stackrel{h_{\boldsymbol{v}}}{\rightarrow} \quad 0^{-}={ }_{\mathrm{Na}^{+}}{ }^{\mathrm{Fe}^{3+}}{ }^{0}\end{array}$ & \\
\hline
\end{tabular}

TABLE IV

Proposed mechanisms for the creation and optical absorption of the minority iron colour centre in smoky quartz

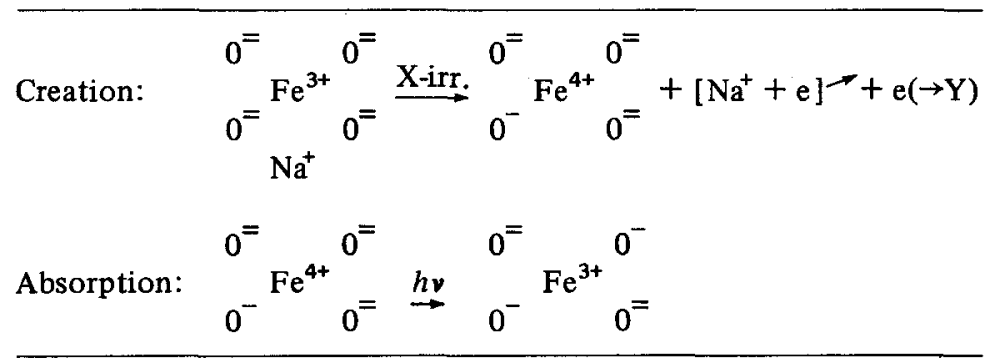

$\mathrm{eV}(390 \mathrm{~nm})$ at room temperature in sample $\mathrm{O}-11$ (fig. 4). This peak is absent at $78 \mathrm{~K}$ and $8 \mathrm{~K}$, suggesting that it should not be attributed to the iron centre but to another centre, presumably in a thermally excited state. As a matter of fact we presume that it should be ascribed to the aluminium centre in its thermally excited state, i.e. in which the hole occupies a $O(3)$ or $O(4)$ position. We already pointed out (section 6.1) that in this state the aluminium centre must indeed exhibit an electromodulation of the absorption.

Acknowledgement. We wish to thank Professor Dr. H. W. de Wijn for reading the manuscript and $\mathrm{Mr}$. A. G. Tijmensen for his assistance with the experiments. The investigations were performed as part of the research programme of the "Stichting voor Fundamenteel Onderzoek der Materie" (F.O.M.) with financial support from the "Nederlandse Organisatie voor Zuiver Wetenschappelijk Onderzoek" (Z.W.O.). 


\section{REFERENCES}

1) Narayanamurti, V. and Pohl, R. O., Rev. mod. Physics 42 (1970) 201.

2) Smoluchowski, R., Proceedings of the XVth Colloque Ampère, (Grenoble, 1968).

3) Bosman, A. J. and Van Daal, H. J., Adv. Physics 19 (1970) 1.

4) Dominik, L. A. K. and MacCrone, R. K., Phys. Rev. 163 (1967) 757.

5) Griffiths, J. H. E., Owen, J. and Ward, I. M., Defects in Crystalline Solids, Report of the Bristol Conference (1954) p. 81.

6) Volger, J. and Stevels, J. M., Philips Res. Rept. 17 (1962) 283.

7) Taylor, A. L. and Farnell, G. W., Canad. J. Phys. 42 (1964) 595.

8) Kuhn, U. and Lüty, F., Solid State Commun. 2 (1964) 281.

9) Austin, I. G., Gamble, R. and Koffyberg, F. P., Solid State Commun. 10 (1972) 1065.

10) Mackey, Jr., J. H., J. chem. Phys. 39 (1963) 74.

11) Mackey, J. H., Boss, J. W. and Wood, D. E., J. Magn. Resonance 3 (1970) 44.

12) Schnadt, R. and Schneider, J., Phys. Kondens. Materie 11 (1970) 19.

13) O’Brien, M. C. M., Proc. Roy. Soc. A231 (1955) 404.

14) Ditchburn, R. W., Mitchell, E. W. J., Paige, E. G. S., Custers, J. F., Dyer, H. B. and Clark, C. D., see ref. 5, p. 92.

15) Cohen, A. J., J. chem. Phys. 25 (1956) 908.

16) Tsinober, L. I. and Chentsova, L. G., Sov. Phys. Crystallography 8 (1963) 214.

17) De Vos, W. J. and Volger, J., Physica 47 (1970) 13.

18) e.g., Schnadt, R. and Räuber, A., Solid State Commun. 9 (1971) 159.

19) See e.g. Fröhlich, H., Theory of Dielectrics, University Press (Oxford, 1958).

20) Tsinober, L. I., Samoilovich, M. I., Gordienko, L. A. and Chentsova, L. G., Sov. Phys. Crystallography 12 (1967) 53.

21) For instance: Schlesinger, M. and Cohen, A. J., J. chem. Phys. 44 (1966) 3146. Chentsova, L. G., Tsinober, L. I. and Samoilovich, M. I., Sov. Phys. Crystallography 11 (1966) 219.

22) Lehmann, G. and Moore, W. J., Science 152 (1966) 1061.

23) Lehmann, G., Z. Naturforschg. 22a (1967) 2080.

24) Barry, T. I., McNamara, P. and Moore, W. J., J. chem. Phys. 42 (1965) 2599.

25) See also Matarrese, L. M., Wells, J. S. and Peterson, R. I., J. chem. Phys. 50 (1969) 2350.

26) E.g., Lehmann, G., Phys. Kondens. Materie 13 (1971) 297.

27) Weeks, R. A., Phys. Rev. 130 (1963) 570.

28) Feigl, F. J. and Anderson, J. H., J. Phys. Chem. Solids 31 (1970) 575.

29) Anderson, J. H. and Weil, J. A., J. chem. Phys. 31 (1959) 427. Haven, Y., Kats, A. and Van Wieringen, J. S., Philips Res. Repts. 21 (1966) 446.

30) Mitchell, E. W. J. and Whitehouse, J. E., J. Phys. Chem. Solids 28 (1967) 1.

31) E.g., Faughnan, B. W.. and Kiss, Z. J., Phys. Rev. Letters 21 (1968) 1331. Ensign, T. C. and Stokowski, S. E., Phys. Rev. B 1 (1970) 2799. 